\title{
Activity and safety of pegylated liposomal doxorubicin, 5-fluorouracil and folinic acid in inoperable hepatocellular carcinoma: A phase II study
}

Giuseppe Di Lorenzo, Antonio Rea, Chiara Carlomagno, Stefano Pepe, Giovannella Palmieri, Roberto Labianca, Antonio Chirianni, Alfonso De Stefano, Vincenzo Esposito, Sabino De Placido, Vincenzo Montesarchio

Giuseppe Di Lorenzo, Chiara Carlomagno, Stefano Pepe, Giovannella Palmieri, Alfonso De Stefano, Sabino De Placido, Cattedra di Oncologia Medica, Dipartimento di Endocrinologia e Oncologia Molecolare e Clinica, Università degli Studi Federico II, Naples, Italy

Antonio Rea, Vincenzo Montesarchio, Divisione di Oncologia, Azienda Ospedaliera Cotugno, Naples, Italy

Antonio Chirianni, Vincenzo Esposito, Divisione di Malattie Infettive Azienda Ospedaliera, Cotugno, Napoli, Italy

Roberto Labianca, Oncology Section, Ospedali Riuniti, Bergamo, Italy

Correspondence to: Giuseppe Di Lorenzo, MD, PhD, Cattedra di Oncologia Medica, Dipartimento di Endocrinologia e Oncologia Molecolare e Clinica, Università degli Studi Federico II , Naples, Italy. giuseppedilorenzoncol@hotmail.com

Telephone: +39-81-7462053 Fax: +39-81-7463660

Received: July 17, 2007 Revised: August 6, 2007

\begin{abstract}
AIM: To improve the results of New therapeutic strategies in hepatocellular carcinoma (HCC). We have conducted a phase II study with pegylated liposomal doxorubicin (PLD), 5-fluorouracil (5FU) and folinic acid (FA).

METHODS: Thirty-one patients with hystologicallyconfirmed, inoperable HCC, received combination chemotherapy with PLD $25 \mathrm{mg} / \mathrm{mq}$ on d 1, 5FU $1200 \mathrm{mg} / \mathrm{mq}$ in $48 \mathrm{~h}$ continuous infusion, and oral FA $30 \mathrm{mg}$ on $\mathrm{d} 1$ and 2 every 3 wk until disease progression or intolerable toxicity.
\end{abstract}

RESULTS: The median age was 65 years (range 41-82) and 28 patients were hepatitis $C$ virus seropositive $(90 \%)$. The majority of patients were Child-Pugh Class B (55\%). Two patients showed a partial response (PR), and 16 had stable disease (SD). With a median follow-up of $14 \mathrm{mo}$, the median time to progression of all evaluable patients was $4 \mathrm{mo}$ (95\% CI 1.7-7). Median overall survival was 9 mo (95\% CI 3-24 mo). After 1 year, 9 of 18 PR/SD patients were alive. Chemotherapy was well tolerated.

CONCLUSION: PLD/FU/FA combination seems capable of achieving durable stabilization of HCC. The manageable toxicity supports a role for combination with other anticancer agents. (c) 2007 WJG. All rights reserved.

Key words: Pegylated liposomal doxorubicin; 5-fluorouracil; Folinic acid; Hepatocellular carcinoma

Di Lorenzo G, Rea A, Carlomagno C, Pepe S, Palmieri G, Labianca R, Chirianni A, De Stefano A, Esposito V, De Placido $S$, Montesarchio V. Activity and safety of pegylated liposomal doxorubicin, 5-fluorouracil and folinic acid in inoperable hepatocellular carcinoma: A phase II study. World J Gastroenterol 2007; 13(48): 6553-6557

http://www.wjgnet.com/1007-9327/13/6553.asp

\section{INTRODUCTION}

Upward trends in incidence or mortality of hepatocellular carcinoma (HCC) have been reported in the United States, Japan and several European countries, including Italy, over the last two or three decades. This increase has been chiefly attributed to the spread of hepatitis $\mathrm{C}$ virus (HCV), which occurred earlier in Japan and Southern Europe than in the United States ${ }^{[1]}$.

Surgical resection and liver transplantation are considered the only cures for HCC, but benefit only about $15 \%$ of patients ${ }^{[2]}$. Patients with unresectable or metastatic disease have a median survival of a few months ${ }^{[3]}$.

It is estimated that about $60 \%$ of all patients with HCC have underlying chirrosis. In some patients, chirrosis associated with portal hypertension and thrombocytopenia makes systemic chemotherapy extremely difficult and contributes to the poor prognosis associated with $\mathrm{HCC}^{[4]}$.

Free doxorubicin has been shown to be superior to symptomatic treatment of $\mathrm{HCC}^{[5]}$, although a meta-analysis has revealed no efficacy of free doxorubicin for treatment of $\mathrm{HCC}^{[6]}$. Pegylated liposomal doxorubicin (PLD) is a novel formulation with markedly delayed clearance, decreased volume of distribution, and accumulation of the drug in malignant tissues, which results in both higher therapeutic efficacy and reduced toxicity ${ }^{[7]}$. A number of studies have shown, at best, response rates of $10 \%-17 \%$, with different schedules and a well-tolerated profile ${ }^{[8-10]}$.

5-Fluorouracil $(5 \mathrm{FU})$ has demonstrated a modest response rate in $\mathrm{HCC}^{[11]}$. There are no published studies 
with combination of PLD and 5FU in HCC. Also, if both drugs show poor activity when used alone, it can be expected that activity will be better when they are used in combination.

We planned a phase II study of PLD, 5FU and FA in patients with inoperable HCC. Our schedule was based on a previous phase I study in upper gastrointestinal cancer ${ }^{[12]}$.

\section{MATERIALS AND METHODS}

\section{Patients}

Patients who were referred to two Italian Departments of Medical Oncology (Cotugno Hospital and Federico II University, Naples) because of their metastatic and/or recurrent HCC were considered eligible for study entry. Main eligibility criteria are shown in Table 1. Patients were required to have histologically confirmed HCC, WHO Performance Status < 2, Stage III or IV, Child-Pugh class $\mathrm{A}$ or $\mathrm{B}$, and no prior systemic chemotherapy. Other inclusion criteria included an absolute neutrophil count of at least 1500 cells per cubic millimeter and/or at least 100000 cells per cubic millimeter, adequate renal (serum creatinine level $<2 \mathrm{mg} / \mathrm{dL}$ ) and cardiac (left ventricular ejection fraction $>50 \%$ by echocardiography) function, and signed written informed consent. The study protocol was reviewed and approved by institutional review board.

\section{Treatment}

Patients receive PLD (Caelyx; Schering-Plough, Milan, Italy) $25 \mathrm{mg} / \mathrm{mq}$ in $500 \mathrm{~mL} \mathrm{5 \%}$ dextrose i.v. over $1 \mathrm{~h}$ on d 1. 5FU (Teva Pharma Italia S.r.L., Milan, Italy) $1200 \mathrm{mg} / \mathrm{mq}$ was administered for $48 \mathrm{~h}$ continuous infusion, and oral FA (Folaren; Ist. Chim. Inter., Rende, Rome, Italy) $30 \mathrm{mg} \mathrm{d} 1$ and 2. Each cycle was given every $3 \mathrm{wk}$. All patients received a standard supportive regimen consisting of hydration and antiemetics. No prophylactic administration of hematopoietic growth factors was allowed. Treatment was repeated until disease progression or unacceptable toxicity. Retreatment occurred upon recovery of platelets to $>100000$ cells per cubic millimeter, neutrophil count of at least 1500 cells per cubic millimeter and skin toxicity or stomatitis to grade $\leqslant 1$. A $25 \%$ dose reduction was permitted for delayed recovery from toxicity of grade $\geqslant 2$. Patients were withdrawn from the study if grade 3-4 hematologic toxicity persisted for more than $2 \mathrm{wk}$.

\section{Response and toxicity evaluation}

Baseline examinations were: complete history and examination, electrocardiography, echocardiography, complete blood count, serum alphafetoprotein, weighted magnetic resonance imaging (MRI) of the liver, abdominal ultrasound, and chest X-ray. If MRI was not feasible, computed tomography was used. Response and toxicity were evaluated every three cycles.

The following WHO criteria were used: (1) a complete response (CR) was defined as the disappearance of all radiological and clinical evidence of tumor, and the absence of all tumor-related symptoms for at least $4 \mathrm{wk}$; (2) a partial response (PR) was defined as a decrease
Table 1 Main eligibility criteria

Age $<85$ yr

WHO performance status 0 or 1

Histologically confirmed HCC

Stage III-IV

No prior systemic chemotherapy

Child-Pugh A or B

Complete history of the patient (including previous treatment)

Informed consent

Normal hematologic, renal and cardiac functions

of $\geqslant 50 \%$ in the product of the largest perpendicular dimensions of measurable tumors, measured at least $4 \mathrm{wk}$ apart; (3) stable disease (SD) was defined as no significant change in radiologic tumor measurements, without worsening in performance status; and (4) progressive disease (PD) was defined as a decline in performance status, the appearance of new malignant lesions, and an increase of $\geqslant 25 \%$ in measurable disease. Toxicity was based on the National Cancer Institute Common Toxicity Criteria version 2.

\section{Statistical analysis}

The primary end-point was the response rate and the secondary measures were survival and time to progression. Sample size was calculated to reject a $10 \%$ response rate in favor of a target response rate of $30 \%$, with a significance level of 0.05 and a power of $80 \%$ by using Simon's optimal two-stage design ${ }^{[13]}$. In the initial stage, a total of 10 evaluable patients were entered and evaluated for response. If more than one response were observed in the first stage, then 21 additional patients were entered in the second stage to achieve a sample size of 31 evaluable patients. Survival rates and time to progression were assessed by the Kaplan-Meier method. All $P$ values were two-sided, with $P$ $<0.05$ indicating statistical significance.

\section{RESULTS}

A total of 31 patients were included in the study between March 2004 and December 2006. Patient characteristics are summarized in Table 2 . The median age was 65 years (range 41-82), and 14 and 17 patients were Child-Pugh class $\mathrm{A}$ and $\mathrm{B}$, respectively. The majority of patients (20) had received prior antitumor therapy. The most common previous treatment for HCC was transarterial chemoembolization, which was received by 15 of 31 patients. Twenty-eight patients were HCV seropositive and 22 showed an alphafetoprotein (AFP) level at the upper limit of normal. Fifteen and 16 patients were in stage IIIC and IV, respectively, according to the TNM system.

\section{Treatment outcomes}

The results are shown in Table 3. Two PR were obtained, and 16 had SD. Sixteen and 8 patients received 6 and 8 treatment cycles, respectively, while 2 were treated with 10 cycles. Among the 16 patients with SD, we observed 8 each in Child-Pugh A and B class. No differences in $\mathrm{PR} / \mathrm{SD}$ were noted between patients who were pretreated 


\section{Table 2 Patient characteristics}

\begin{tabular}{lc}
\hline No. of patients & 31 \\
Male & 26 \\
Female & 5 \\
Median age (range) & $65(41-82) \mathrm{yr}$ \\
WHO performance status & 12 \\
0 & 19 \\
1 & \\
Positive hepatitis status & 3 \\
Hepatitis B & 28 \\
Hepatitis C & 22 \\
AFP > ULN & 9 \\
Yes & \\
No & 14 \\
Child-Pugh status & 17 \\
A & \\
B & 15 \\
Disease stage at study entry (TNM) & 16 \\
III C & \\
IV & 15 \\
Grading (AJCC) at initial diagnosis & 10 \\
Well-differentiated & 6 \\
Moderately-well-differentiated & \\
Poorly differentiated & 15 \\
Prior treatment & 6 \\
Chemoembolization & 5 \\
Local alcholization & 10 \\
Local radiofrequency & \\
None & \\
\hline
\end{tabular}

Data are expressed as median (range) or $n$. WHO: World Health Organization. ULN: Upper limit of normal; AJCC: American Joint Committee on Cancer.

and those who were not, such as those in stage IIIC and IV. With a median follow-up of $14 \mathrm{mo}$, the median time to progression of all evaluable patients was 4 mo $(95 \%$ CI 1.7-7). Median overall survival was 9 mo (95\% CI 3-24 mo). Among 18 responder/SD patients, $9(50 \%)$ were alive at $12 \mathrm{mo}$, while at the same time, none of the PD patients were alive $(P<0.05)$. One responder who received 10 cycles of chemotherapy was alive at 24 mo. Of 31 evaluable patients, $16(52 \%)$ showed a $>50 \%$ decrease in serum AFP from baseline. Among 13 patients with PD, 5 progressed to Child-Pugh C class. After disease progression, 6 patients had salvage treatment with platinum compound or thalidomide, while 25 patients received only supportive care.

\section{Safety}

In total, 130 cycles of chemotherapy were administered, with a median of 6 cycles (range 2-10), and 13 of the planned cycles were delayed because of toxic effects. Dose reduction was required in 20 cycles. All eligible patients were evaluable for toxic effects (Table 4). The most frequent toxic effects were neutropenia grade 1-2 and oral stomatitis in 7 patients each. These effects were well managed and did not require treatment discontinuation. Grade 3-4 anemia, neutropenia and thrombocytopenia were observed in 2, 3 and 2 patients, respectively. These side effects required hematopoietic growth factors and red-cell transfusion during the treatment. Hand-foot skin rash occurred in 5 patients and was limited to low-grade intensity.
Table 3 Responses and survival rates $(n=31)$

\begin{tabular}{lc}
\hline Response after three cycles & No. of patients $(\%)$ \\
PR & $2(6.5)$ \\
SD & $16(51.5)$ \\
Progression & $13(42)$ \\
Median progression-free survival $(95 \% \mathrm{CI})$ & $4(1.7-7) \mathrm{mo}$ \\
Overall survival rate & \\
$6 \mathrm{mo}$ & $21(68)$ \\
$12 \mathrm{mo}$ & $9(29)$ \\
$24 \mathrm{mo}$ & $1(0.3)$ \\
Survival rate in & \\
PR or SD patients $(n=18)$ & \\
6 mo & $15(83)$ \\
$12 \mathrm{mo}$ & $9(50)$ \\
$24 \mathrm{mo}$ & $1(0.56)$ \\
\hline
\end{tabular}

Table 4 Reported toxicity by 31 patients $n(\%)$

\begin{tabular}{lll}
\hline Toxicity & Grade 1-2 & Grade 3-4 \\
\hline Neutropenia & $7(22.5)$ & $3(10)$ \\
Anemia & $8(26)$ & $2(6.5)$ \\
Thrombocytopenia & $5(16)$ & $2(6.5)$ \\
Oral stomatitis & $7(22.5)$ & $1(3)$ \\
Nausea/vomiting & $3(10)$ & $2(6.5)$ \\
Diarrhea & $3(10)$ & $1(3)$ \\
Hand-foot syndrome & $5(16)$ & 0 \\
Hepatic dysfunction & $3(10)$ & 0 \\
Peripheral neuropathy & $3(10)$ & 0 \\
\hline
\end{tabular}

\section{DISCUSSION}

There is no consensus on the treatment of patients with advanced-stage HCC. Locoregional approaches (embolization, intra-arterial chemotherapy and chemoembolization) and systemic treatments have offered marginal survival benefit in most clinical trials ${ }^{[14]}$. The overall response rate to systemic chemotherapy is generally $<10 \%{ }^{[15]}$, probably owing to the strong multidrugresistance gene expression that is usually observed in this setting ${ }^{[16]}$. Doxorubicin has been widely evaluated in patients with advanced stage HCC, either alone or in combination. The combination of cisplatin, doxorubicin, 5FU and alpha-interferon (PIAF) has been assessed in 50 patients. The overall response rate was $26 \%$, but considerable hematological toxicity and 2 treatmentrelated deaths occurred ${ }^{[17]}$. There was an expectation that doxorubicin efficacy would be enhanced with the bettertolerated PLD formulation ${ }^{[18]}$.

A number of studies have shown, with different doses and schedules, at best, response rates of 10\%-17\% with PLD alone ${ }^{[8-10]}$. Two other studies have revealed no response, and with a median survival of $20 \mathrm{wk}$, the authors concluded that PLD was ineffective ${ }^{[19,20]}$. Based on a median survival of 1 year, Schmidinger et a ${ }^{9]}$ concluded that PLD warrented further study; however, Ruff et a ${ }^{[8]}$ achieved a median survival of only 5.3 mo.

5-fluorouracil has been used alone in HCC, with modest activity ${ }^{[11]}$. In an attempt to achieve better effectiveness we have combined PLD and 5FU. To date, there have been no published studies with PLD and 
5FU in HCC. We chose to use PLD $25 \mathrm{mg} / \mathrm{mq}$ and $5 \mathrm{FU} 1200 \mathrm{mg} / \mathrm{mq}$ every $3 \mathrm{wk}$ since we considered, as a result of a previous phase I study ${ }^{[12]}$, that these doses would be well tolerated. In our trial, the combination of PLD, 5FU and FA was well tolerated, and we achieved an overall response rate of $6.5 \%$. However, despite the modest overall response rate, we observed SD in $>50 \%$ of patients. This information is encouraging, and PLD/ FU/FA combination seems capable of achieving durable stabilization of HCC.

Median time to progression was $4 \mathrm{mo}$, which is longer than that in other PLD studies, such as those by Valle et al and Halm et $a{ }^{[19,21]}$. Median overall survival was $9 \mathrm{mo}$ and was shorter than that in the study by Schmidinger $e t a l^{[9]}$, but longer than that in previous PLD studies ${ }^{[8,10,19,21]}$.

Among 18 responder/SD patients, nine (50\%) were alive at $12 \mathrm{mo}$, while at the same time, none of those with PD was alive $(P<0.05)$. Our SD rate $(50 \%)$ was higher than that in the studies by Valle et al and Halm et al. Our study showed better results than those in a recent study by Poh et al, which showed no objective response after treatment with combined PLD and 5FU analogue (capecitabine) ${ }^{[22]}$. Higher PR and SD rates, and longer survival were observed by Louafi et a ${ }^{[23]}$. Thirtyfour patients have been treated with gemcitabine plus oxaliplatin (GEMOX). The overall response rate was $18 \%$ and SD was observed in $58 \%$ of patients. Median overall survival was $11.5 \mathrm{mo}$.

However, these differences could have been due to different selection criteria. The GEMOX trial included few Child-Pugh Class B and pretreated patients compared to our study. It should be kept in mind that the degree of cirrhosis in our patients was not only limited to Child-Pugh class A but also Class B patients. In our trial, the majority of patients were Class B. Other studies have not included Class B patients ${ }^{[24]}$, have not indicated whether they include Class B patients ${ }^{[21]}$, or if this class was included, it was in the minority ${ }^{[19,23]}$. This point strengthens our results. In the past 15 years, about 10 new chemotherapeutic agents have become available, which has led to major advances in the medical treatment of several gastrointestinal tract cancers, such as colon adenocarcinoma. However, recent clinical trials of the taxanes irinotecan and topotecan have all given disappointing results for $\mathrm{HCC}^{[25-27]}$. New targeted antitumoral therapies have been tested in HCC patients over the past 2 years. Preliminary results of a phase III randomized trial have shown that first-line treatment with sorafenib improves survival in HCC when compared to placebo $^{[28]}$.

In conclusion, a major limit of our study was that it was a small phase II study; however, we believe that the results, especially in terms of long-term disease stabilization, merit further evaluation. For example, it would be interesting to carry out additional clinical studies that involve novel targeted agents in combination with cytotoxic agents such as PLD and FUFA. One of the next steps will be to combine this schedule with biological agents in HCC, such as bortezomib, since synergy between bortezomib and anthracyclines has been observed $^{[20]}$.

\section{COMMENTS}

\section{Background}

There is no consensus on the treatment of patients with advanced-stage HCC. Locoregional approaches (embolization, intra-arterial chemotherapy and chemoembolization) and systemic treatments have offered a marginal survival benefit in most clinical trials.

\section{Research frontiers}

The overall response rate to systemic chemotherapy is generally $<10 \%$, probably owing to the strong multidrug-resistance gene expression that is usually observed in this setting.

\section{Related publications}

A number of studies have shown, with different doses and schedules, at best, response rates of $10 \%-17 \%$ with PLD alone. 5FU has been used alone in HCC, with modest activity.

\section{Innovations and breakthroughs}

In an attempt to achieve better effectiveness, we combined PLD and 5FU. To date, there have been no published studies with PLD and 5FU in HCC. In our trial, combination of PLD, 5FU and FA was well tolerated, and we achieved an overall response rate of $6.5 \%$. However, despite the modest overall response rate, we observed SD in $>50 \%$ of patients. Our SD rate is higher than that in the studies of Valle et al and Halm et al. Median time to progression was $4 \mathrm{mo}$, which is higher than that in other PLD studies.

\section{Applications}

It should be kept in mind that the degree of cirrhosis of our patients was not only limited to Child-Pugh class A but also Class B patients. In our trial, the majority of patients were Class B. Other studies did not include Class B patients, did not indicate whether they included Class B patients, or if this class was included, it was in the minority. This point strengthens our results. We believe that our results, especially in terms of long-term disease stabilization, merit further evaluation. It would be interesting to carry out additional clinical studies that involve novel targeted agents, such as bortezomib, in combination with PLD and FUFA.

\section{Terminology}

Pegylated liposomal doxorubicin (PLD) is a novel formulation with markedly delayed clearance, decreased volume of distribution, and accumulation of the drug in malignant tissues, which results in both higher therapeutic efficacy and reduced toxicity.

\section{Peer review}

Although this study had a small sample size, the results are interesting. Inoperable HCC was stabilized with chemotherapy that was well tolerated. In addition, this is believed to be the first report of the use of PLD in combination with 5FU As for reviewer comments, how do the authors explain the modest overall response rate of $6.5 \%$ and yet stabilization of disease in $51.1 \%$ ? Was there any progression in the liver disease or hepatic function tests during the administration of chemotherapy? Did any patients progress to Child-Pugh class $\mathrm{C}$ ?

\section{REFERENCES}

1 La Vecchia C, Lucchini F, Franceschi S, Negri E, Levi F. Trends in mortality from primary liver cancer in Europe. Eur J Cancer 2000; 36: 909-915

2 Llovet JM, Bruix J, Gores GJ. Surgical resection versus transplantation for early hepatocellular carcinoma: clues for the best strategy. Hepatology 2000; 31: 1019-1021

3 Abou-Alfa GK. Hepatocellular carcinoma: molecular biology and therapy. Semin Oncol 2006; 33: S79-S83

4 Nzeako UC, Goodman ZD, Ishak KG. Hepatocellular carcinoma in cirrhotic and noncirrhotic livers. A clinicohistopathologic study of 804 North American patients. Am J Clin Pathol 1996; 105: 65-75

5 Lai CL, Wu PC, Chan GC, Lok AS, Lin HJ. Doxorubicin versus no antitumor therapy in inoperable hepatocellular carcinoma. A prospective randomized trial. Cancer 1988; 62: 479-483 
6 Simonetti RG, Liberati A, Angiolini C, Pagliaro L. Treatment of hepatocellular carcinoma: a systematic review of randomized controlled trials. Ann Oncol 1997; 8: 117-136

7 Gabizon A, Martin F. Polyethylene glycol-coated (pegylated) liposomal doxorubicin. Rationale for use in solid tumours. Drugs 1997; 54 Suppl 4: 15-21

8 Ruff P, Moodley SD, Rapaport DA. Long term follow-up of pegylated liposomal doxorubicin: a well tolerated and effective agent in advanced hepatocellular carcinoma (HCC). Proc Am Soc Clin Oncol 2001; 20: Abstract: 667

9 Schmidinger $\mathbf{M}$, Wenzel C, Locker GJ, Muehlbacher F, Steininger R, Gnant M, Crevenna R, Budinsky AC. Pilot study with pegylated liposomal doxorubicin for advanced or unresectable hepatocellular carcinoma. Br J Cancer 2001; 85: 1850-1852

10 Hong RL, Tseng YL. A phase II and pharmacokinetic study of pegylated liposomal doxorubicin in patients with advanced hepatocellular carcinoma. Cancer Chemother Pharmacol 2003; 51: 433-438

11 Lin DY, Lin SM, Liaw YF. Non-surgical treatment of hepatocellular carcinoma. I Gastroenterol Hepatol 1997; 12: S319-S328

12 Hofheinz RD, Willer A, Weisser A, Gnad U, Saussele S, Kreil S, Hartmann JT, Hehlmann R, Hochhaus A. Pegylated liposomal doxorubicin in combination with mitomycin $\mathrm{C}$, infusional 5 -fluorouracil and sodium folinic acid. A phase-I-study in patients with upper gastrointestinal cancer. Br J Cancer 2004; 90: 1893-1897

13 Simon R. Optimal two-stage designs for phase II clinical trials. Control Clin Trials 1989; 10: 1-10

14 Bruix J, Sherman M, Llovet JM, Beaugrand M, Lencioni R, Burroughs AK, Christensen E, Pagliaro L, Colombo M, Rodés J. Clinical management of hepatocellular carcinoma. Conclusions of the Barcelona-2000 EASL conference. European Association for the Study of the Liver. J Hepatol 2001; 35: 421-430

15 Nowak AK, Chow PK, Findlay M. Systemic therapy for advanced hepatocellular carcinoma: a review. Eur J Cancer 2004; 40: 1474-1484

16 Chou YY, Cheng AL, Hsu HC. Expression of P-glycoprotein and p53 in advanced hepatocellular carcinoma treated by single agent chemotherapy: clinical correlation. J Gastroenterol Hepatol 1997; 12: 569-575

17 Leung TW, Patt YZ, Lau WY, Ho SK, Yu SC, Chan AT, Mok TS, Yeo W, Liew CT, Leung NW, Tang AM, Johnson PJ. Complete pathological remission is possible with systemic combination chemotherapy for inoperable hepatocellular carcinoma. Clin Cancer Res 1999; 5: 1676-1681

18 Uziely B, Jeffers S, Isacson R, Kutsch K, Wei-Tsao D, Yehoshua Z, Libson E, Muggia FM, Gabizon A. Liposomal doxorubicin: antitumor activity and unique toxicities during two complementary phase I studies. J Clin Oncol 1995; 13: 1777-1785

19 Halm U, Etzrodt G, Schiefke I, Schmidt F, Witzigmann H, Mössner J, Berr F. A phase II study of pegylated liposomal doxorubicin for treatment of advanced hepatocellular carcinoma. Ann Oncol 2000; 11: 113-114

20 Miller RL, Bowen KE, Chung HG. A phase II study of liposomal doxorubicin in patients with advanced hepatocellular carcinoma (HCC). Proc Am Soc Clin Oncol 2002; 21: Abstract: 2324

21 Valle JW, Dangoor A, Beech J, Sherlock DJ, Lee SM, Scarffe JH, Swindell R, Ranson M. Treatment of inoperable hepatocellular carcinoma with pegylated liposomal doxorubicin (PLD): results of a phase II study. Br J Cancer 2005; 92: 628-630

22 Poh SB, Bai LY, Chen PM. Pegylated liposomal doxorubicinbased combination chemotherapy as salvage treatment in patients with advanced hepatocellular carcinoma. Am J Clin Oncol 2005; 28: 540-546

23 Louafi S, Boige V, Ducreux M, Bonyhay L, Mansourbakht T, de Baere T, Asnacios A, Hannoun L, Poynard T, Taïeb J. Gemcitabine plus oxaliplatin (GEMOX) in patients with advanced hepatocellular carcinoma (HCC): results of a phase II study. Cancer 2007; 109: 1384-1390

24 Park SH, Lee Y, Han SH, Kwon SY, Kwon OS, Kim SS, Kim JH, Park YH, Lee JN, Bang SM, Cho EK, Shin DB, Lee JH. Systemic chemotherapy with doxorubicin, cisplatin and capecitabine for metastatic hepatocellular carcinoma. BMC Cancer 2006; 6: 3

25 Chao Y, Chan WK, Birkhofer MJ, Hu OY, Wang SS, Huang YS, Liu M, Whang-Peng J, Chi KH, Lui WY, Lee SD. Phase II and pharmacokinetic study of paclitaxel therapy for unresectable hepatocellular carcinoma patients. Br J Cancer 1998; 78: 34-39

26 O'Reilly EM, Stuart KE, Sanz-Altamira PM, Schwartz GK, Steger CM, Raeburn L, Kemeny NE, Kelsen DP, Saltz LB. A phase II study of irinotecan in patients with advanced hepatocellular carcinoma. Cancer 2001; 91: 101-105

27 Alexandre J, Tigaud JM, Gross-Goupil M, Gornet JM, Romain D, Azoulay D, Misset JL, Goldwasser F. Combination of topotecan and oxaliplatin in inoperable hepatocellular cancer patients. Am J Clin Oncol 2002; 25: 198-203

28 Llovet J, Ricci S, Mazzaferro V. Sorafenib improves survival in advanced Hepatocellular Cancer. A randomized placebocontrolled trial (SHARP Trial). Proc Am Soc Clin Oncol Chicago 2007, Plenary Session; Abstract: LBA1

29 Mitsiades N, Mitsiades CS, Richardson PG, Poulaki V, Tai YT, Chauhan D, Fanourakis G, Gu X, Bailey C, Joseph M, Libermann TA, Schlossman R, Munshi NC, Hideshima T, Anderson KC. The proteasome inhibitor PS-341 potentiates sensitivity of multiple myeloma cells to conventional chemotherapeutic agents: therapeutic applications. Blood 2003; 101: $2377-2380$

S- Editor Liu Y L- Editor Kerr C E- Editor Wang HF 